\title{
Astragalus polysaccharides mediated preventive effects on bronchopulmonary dysplasia in rats
}

\author{
Xiao-Hong Wang ${ }^{1}$, Hong-Ling Jia ${ }^{2}$, Li Deng ${ }^{3}$ and Wei-Min Huang ${ }^{1}$
}

BACKGROUND: Bronchopulmonary dysplasia (BPD) is a multifactor chronic lung disease that mainly affects premature infants. In this study, we investigate the preventive effects of Astragalus polysaccharides (APS) on BPD, and explore its potential molecular mechanisms.

METHODS: Lung tissues of newborn Sprague-Dawley rats from the control group, the room air plus low-dose APS group, the room air plus high-dose APS group, the BPD model group, the low-dose APS group ( $20 \mathrm{mg} / \mathrm{kg} \mathrm{d})$, and the high-dose APS group (40 $\mathrm{mg} / \mathrm{kg} \mathrm{d}$ ) were examined at the 4th, 10th, and 14th $\mathrm{d}$ of life. The pathomorphological change was evaluated by hematoxylin-eosin staining. The content levels of superoxide dismutase (SOD) and malondialdehyde (MDA) were measured by the assay kit. Moreover, the protein and/or mRNA expression levels of NF-KBp65, CD31, ICAM-1, and TNF-a were also detected by corresponding methods.

RESULTS: APS decreased the inflammatory cells infiltrating compared with the BPD group. For the APS group, the activity of SOD was increased and the content of MDA was reduced compared with the BPD group at any time point. The protein and mRNA expression levels of NF-KBp65, ICAM-1, and TNF- $a$ were all decreased, while the protein expression level of CD31 was increased in the APS-treated group, with the most significant difference of the high-dose group $(P<0.01)$ compared with the BPD group after birth on the 4th, 10th, and 14th $\mathrm{d}$.

CONCLUSION: APS can reduce airway remodeling and alveolar damage by its modulation of inflammatory mediators and antioxidation, suggesting some protective effects on BPD of neonatal rats.

B ronchopulmonary dysplasia (BPD) is a multifactor chronic lung disease, which is characterized by simplified alveolarization and arrested vascular development of the lung with associated evidence of endothelial dysfunction, inflammation, and increased oxidative damage that continues to affect premature infants despite advances in clinical care $(1,2)$.

Although the exact etiology and pathophysiology are complex and still remain unclear, recent studies do support the roles for oxidative stress and inflammation-mediated damage, which including reactive oxygen species (ROS) and cytokines contribute to the inflammatory cascade in modulating the immune system of BPD. The proinflammatory cytokines, such as tumor necrosis factor-alpha (TNF- $\alpha$ ), interleukin-1 $\beta$ (IL-1 $\beta$ ), and interleukin-6 (IL-6) in the serum and tracheal aspirate, were significantly increased. However, the anti-inflammatory cytokines, such as interleukin-10 (IL-10), were significantly decreased in BPD (3). Moreover, TNF- $\alpha$ has overlapping and synergetic activities to induce the production of nuclear factor- $\mathrm{KB}$ (NF- $\mathrm{KB})$ (4). Although steroids could inhibit inflammatory mediators through various mechanisms, the adverse effects on growth and development limit their use $(5,6)$. Therefore, the therapeutic tool of BPD still remains an important unsolved problem.

APS is an extract from the Chinese traditional herb Astragalus membranaceus, which has strong antioxidant and anti-inflammatory properties by enhancing the activity of antioxidant enzymes in the blood and reducing the level of some organizations lipid peroxide lipid peroxide in kidney, heart, and so on (7). In this study, we used it to gain an insight on its role in the anti-inflammation mechanism of reactive oxygen species (ROS) and cytokine regulation for the development of BPD.

\section{RESULTS}

\section{Preventive Effect of APS for Histological Damage}

Lung histological damage was associated with oxidative stress and inflammation, which was examined by hematoxylin and eosin staining. From this study, we know that there was no damage in control groups at the 10th and 14th d for SpragueDawley (SD) newborn rats. It was not significantly different from both the room air (RA) plus low-dose group (RA+LD APS group) and the high-dose APS group (RA+HD APS group) compared with the control group.

However, lung tissues showed cell infiltration, and alveolar and vascular development arrested in the BPD group at the 10th and 14th $\mathrm{d}$. The damage type of lung histology was fewer and larger simplified alveoli, negligible airway lesions, variable interstitial fibroproliferation, fewer and dysmorphic capillaries, and less severe arterial lesions.

But in the APS treatment group, the extent of inflammation, the severity of lung damage, and alveolar and lung vascular 


\section{Articles Wang etal.}

development were significantly improved in a dose-dependent manner at the 10th and 14th $\mathrm{d}$. The mean linear intercept (MLI) in the APS-treated group was significantly low and the mean alveolar number (MAN) per square area was significantly high compared with the time-matched BPD group $(P<$ 0.05 or $P<0.01$ ), and there was no significant difference in the lung volume between each group at the corresponding time points (Table 1). This mainly reflected the preventive effect that inhibited the extent of inflammation, prevented alveolar injury, and attenuated arrested alveolar growth (Figure 1).

\section{Effect of APS Modulation on the Level of MDA and the Activity of SOD}

The levels of MDA were often used as an indication of oxidative damage and as a marker for free-radical-induced lipid peroxidation (8). The known function of SOD is to convert toxic superoxide radicals to potentially less toxic hydrogen peroxide and water (9). Lung injury might be well controlled by the administration of exogenous antioxidants and recombinant human SOD (rhSOD) $(10,11)$. From this study, the activity of SOD significantly decreased and the content of MDA significantly increased compared with the control group at the 14th $d$ in the BPD group. For the HD APS group, the activity of SOD was increased and the content of MDA was reduced compared with the BPD group at any time point, while this result was only seen at the 10th $d$ on the LD APS group (Table 2). There was no significant difference for the activity of SOD and the content of MDA in the RA plus highand low-dose APS group compared with the control group at any time point.

Table 1. Histomorphologic analysis of rat lung tissues treated with or without APS

\begin{tabular}{|c|c|c|c|c|c|c|}
\hline \multirow[b]{2}{*}{ Group } & \multicolumn{2}{|c|}{$V_{\mathrm{L}}(\mathrm{ml})$} & \multicolumn{2}{|c|}{$\mathrm{MLI}(\mu \mathrm{m})$} & \multicolumn{2}{|c|}{$\operatorname{MAN}\left(/ 10^{4} \mu \mathrm{m}^{2}\right)$} \\
\hline & $10 d$ & $14 d$ & $10 d$ & $14 d$ & $10 d$ & $14 d$ \\
\hline Control & $0.205 \pm 0.013$ & $0.287 \pm 0.016$ & $33.10 \pm 3.66$ & $32.78 \pm 1.99$ & $33.23 \pm 1.88$ & $36.36 \pm 5.20$ \\
\hline BPD & $0.198 \pm 0.019$ & $0.284 \pm 0.011$ & $37.49 \pm 0.67^{\dagger}$ & $37.53 \pm 2.41^{\dagger}$ & $21.84 \pm 1.67^{\ddagger}$ & $25.42 \pm 1.83^{\dagger}$ \\
\hline HD APS & $0.203 \pm 0.022$ & $0.285 \pm 0.013$ & $31.64 \pm 1.98^{*}$ & $30.92 \pm 1.85^{*}$ & $34.44 \pm 2.01^{* *}$ & $39.94 \pm 4.71^{*}$ \\
\hline
\end{tabular}

Values are means \pm SD. Data were analyzed by one-way ANOVA and post hoc Newman-Keuls analysis.

APS, Astragalus polysaccharides; BPD, bronchopulmonary dysplasia; HD, high-dose; MAN, mean alveolar number; MLI, mean linear intercept; RA; room air; $V_{\downarrow}$, left lung volume

*Significantly different from the time-matched BPD group $(P<0.05)$. ${ }^{* *}$ Significantly different from the time-matched BPD group $(P<0.01)$. ${ }^{*}$ Significantly different from the timematched control group $(P<0.05)$. ${ }^{\ddagger}$ Significantly different from the time-matched control group $(P<0.01) ; n=8$ separate samples.

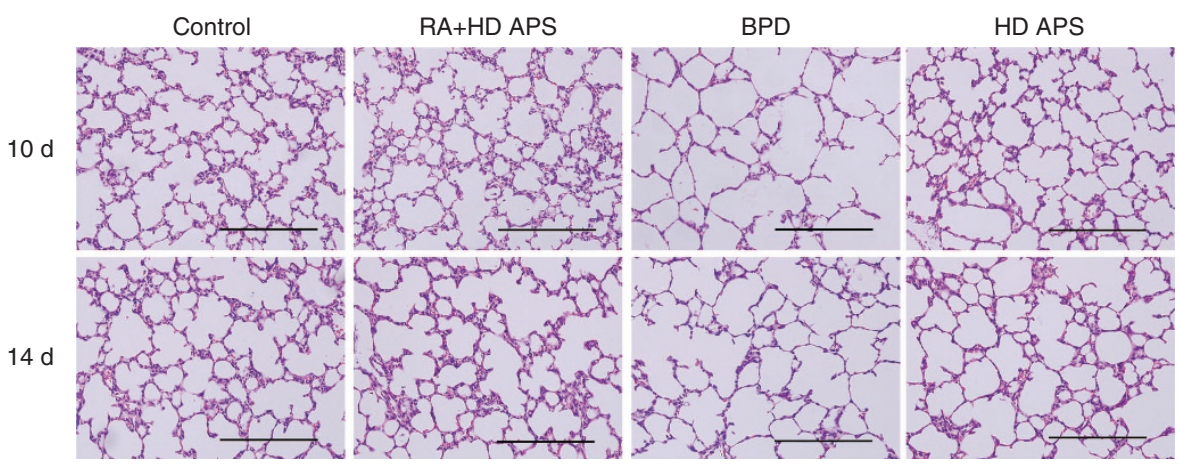

Figure 1. Hematoxylin and eosin staining of lung tissues. (Scale bars $=100 \mu \mathrm{m}$; magnification: $\times 200$. The low-dose APS group is not shown.) APS, Astragalus polysaccharides; BPD, bronchopulmonary dysplasia; HD, high-dose; RA, room air.

Table 2. SOD activity and MDA levels of rat lung tissues treated with or without APS

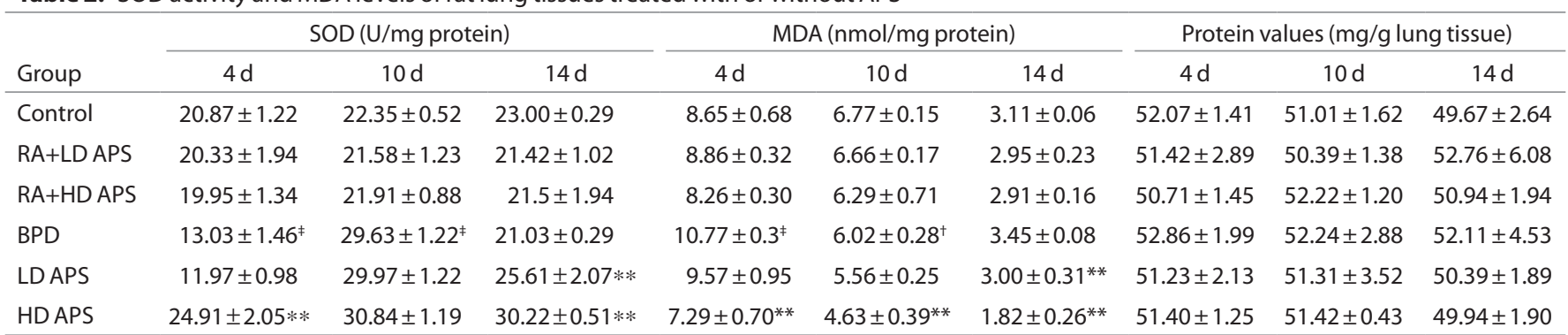

Values are means \pm SD. Data were analyzed by one-way ANOVA and post hoc Newman-Keuls analysis.

APS, Astragalus polysaccharides; BPD, bronchopulmonary dysplasia; HD, high-dose; LD, low-dose; MDA, malondialdehyde; RA, room air; SOD, superoxide dismutase. **Significantly different from the time-matched BPD group $(P<0.01)$.; ${ }^{\dagger}$ Significantly different from the time-matched control group $(P<0.05) . ;$ Significantly different from the time-matched control group $(P<0.01) ; n=8$ separate samples. 
mRNA Levels of NF-кBp65, Intercellular Cell Adhesion Molecule-1, and TNF- $\alpha$ in Lung Tissues Identified by Real-Time PCR

Real-time PCR analysis showed that the intercellular cell adhe-

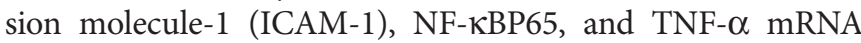
expression levels of the BPD group were significantly higher than the control group at each time point $(P<0.05)$, while the mRNA expression level was significantly reduced in the APS groups in a dose-dependent manner at each time point $(P<$ $0.001)$. The maximum inhibition effect was mainly seen in the HD APS group (Figure 2). No significant differences were seen among the control group, the RA plus high-dose APS group, and the low-dose APS group at any time point for the newborn rats.

\section{Significantly Decreased TNF- $\alpha$ Protein Expression by the Effect} of APS

The positive cells of TNF- $\alpha$ mainly located in interstitial lung with brown-yellow cytoplasm and negative control show the absence of staining. There was almost no TNF- $\alpha$ protein expression in the control group at the 10th $\mathrm{d}$ and little TNF- $\alpha$ protein expression at the 14th $\mathrm{d}$, and no apparent expression in the RA plus high-dose and low-dose APS groups at the 10th and 14th $\mathrm{d}$. The expression of TNF- $\alpha$ in the BPD model group significantly increased compared with the control group, which was overexpressed at both the 10th and 14th $\mathrm{d}$. The expression of TNF- $\alpha$ in the APS group was significantly decreased in a dose-dependent manner compared with the BPD model group

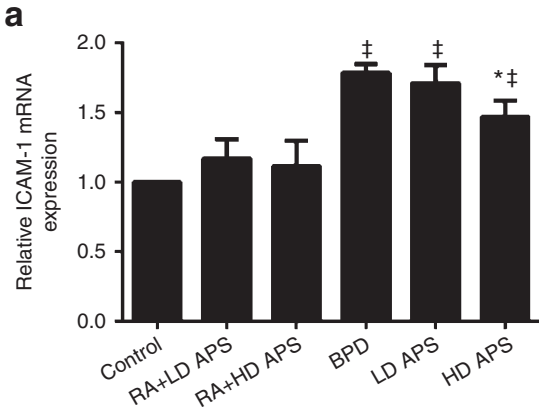

$4 \mathrm{D}$
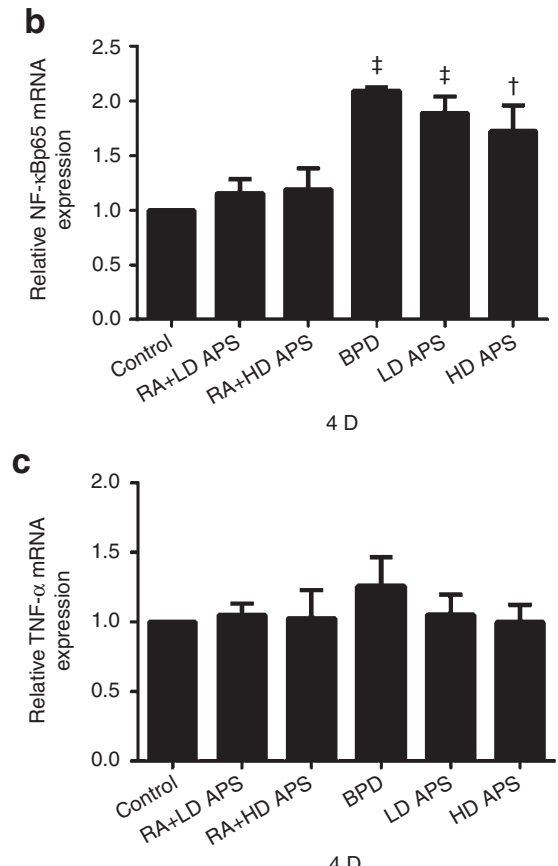

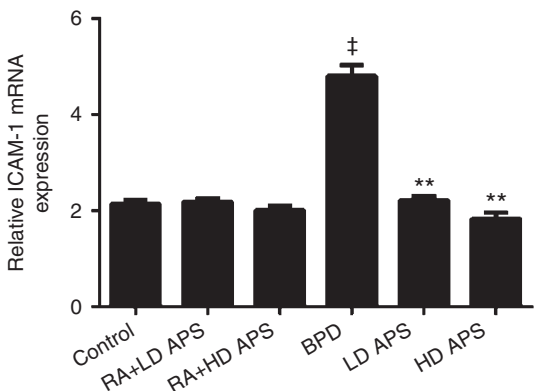

$10 \mathrm{D}$

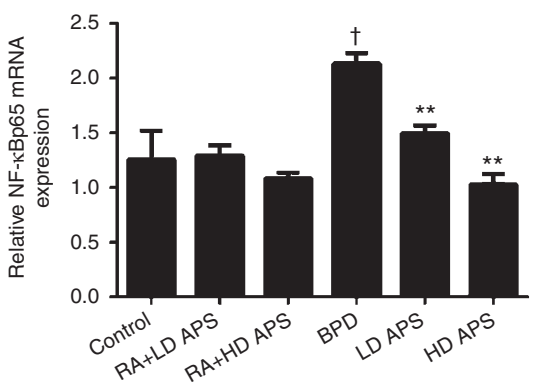

$10 \mathrm{D}$

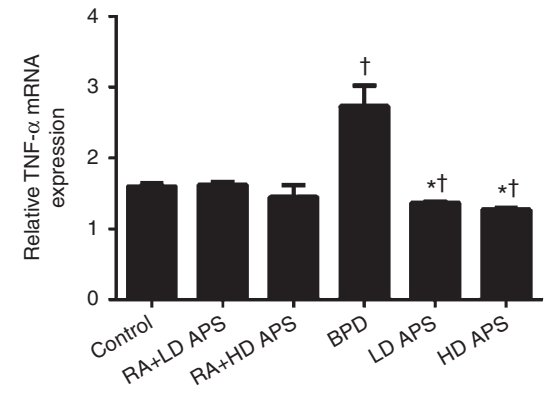

$10 \mathrm{D}$

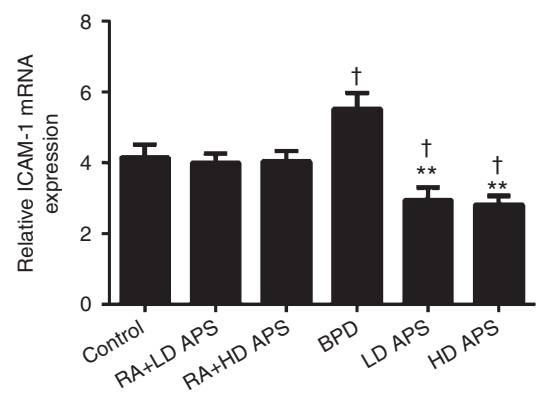

$14 \mathrm{D}$

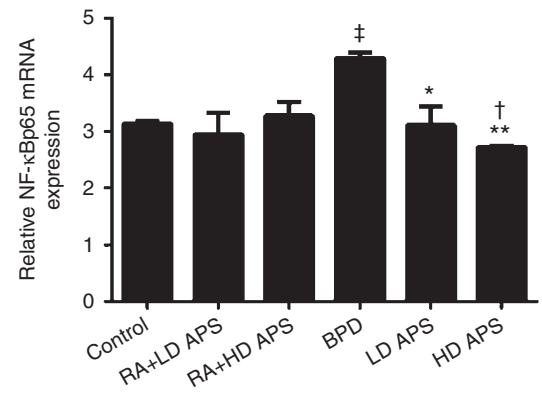

$14 \mathrm{D}$

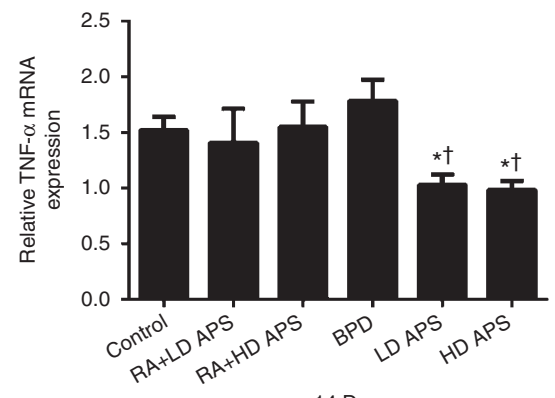

$14 \mathrm{D}$

Figure 2. Effects of Astragalus polysaccharides (APS) on intercellular cell adhesion molecule-1 (ICAM-1), NF-KBp65, and tumor necrosis factor-alpha (TNF- $\alpha$ ) mRNA expression in neonatal rat lungs. Total RNA was isolated from the neonatal rat lung. The mRNA expression was measured by $2^{-\triangle \Delta C T}$. (a) mRNA expression of ICAM-1 of the control group, the room air (RA) plus low-dose APS group, the RA plus high-dose APS group, the bronchopulmonary dysplasia (BPD) model group, the low-dose APS group, and the high-dose APS group. (b) mRNA expression of NF- $\mathrm{kBp} 65$ of the control group, the RA plus low-dose APS group, the RA plus high-dose APS group, the BPD model group, the low-dose APS group, and the high-dose APS group. (c) mRNA expression of TNF- $\alpha$ of the control group, the RA plus low-dose APS group, the RA plus high-dose APS group, the BPD model group, the low-dose APS group, and the high-dose APS group. Values are means $\pm \mathrm{SD}(n=8)$. *Significantly different from the time-matched BPD group $(P<0.05)$, **significantly different from the time-matched BPD group $(P<0.001)$. 'Significantly different from the time-matched control group $(P<0.05)$, ${ }^{\ddagger}$ significantly different from the time-matched control group $(P<0.001)$. 
at the 4th (not shown), 10th, and 14th $\mathrm{d}$ and the changes were significantly apparent in the HD APS group (Figure 3).

\section{Significant Increase of Platelet Endothelial Cell Adhesion Molecule-1 Protein Expression and Vascular Density by the Effect of APS}

Cell adhesion molecule-1 (CD31) protein was mainly expressed in the cytoplasm of pulmonary microvascular endothelial cells. Both CD31 and vascular density in the BPD group were decreased compared with the control group. The expression of CD31 and vascular density in the APS group was significantly increased in a dose-dependent manner compared with the BPD model group and the changes were significantly apparent in the HD APS group $(P<0.05$ or $P<0.01)$ (Figure 4 and Table 3).

\section{Effect of APS on the Neutrophil and Macrophage Counts in Bronchoalveolar Lavage Fluid}

From this study, both the number of neutrophils and macrophages were significantly increased in the BPD group compared with the control group at any time point $(P<0.01$, Table 4). The counts of neutrophils and macrophages were significantly decreased in APS groups compared with the BPD group $(P<0.05$ or $P<0.01)$. No significant differences were apparent between the control group and the room air plus APS group at any time point.

\section{Expression of NF-KBp65 and ICAM-1 in Lung Tissues Identified by Western Blot}

The expressions of NF- $\mathrm{KBp} 65$ and ICAM-1 in lung tissues from each pup group were examined at the 4 th, 10th, and 14 th
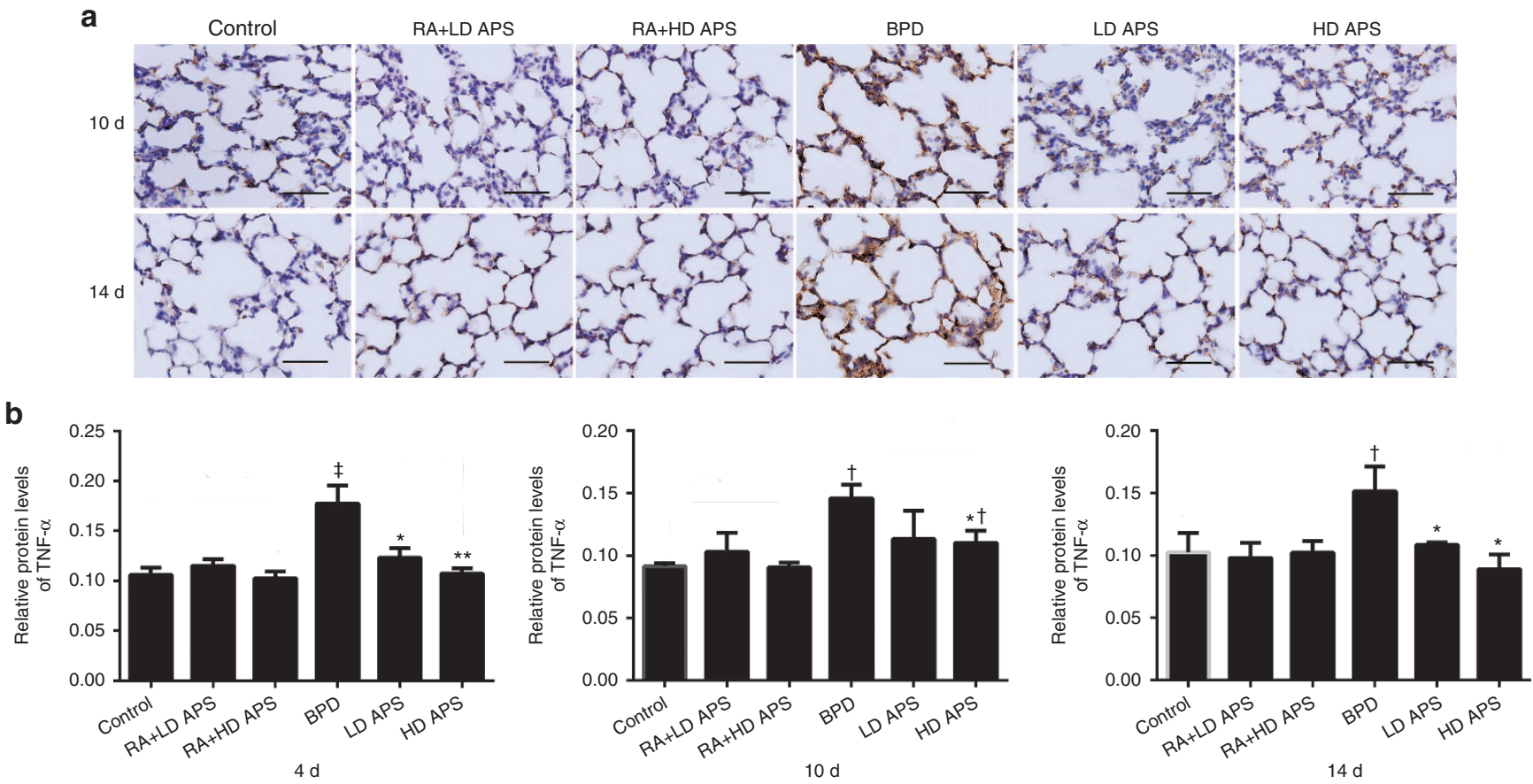

Figure 3. Effects of Astragalus polysaccharides (APS) on TNF-a protein expression in neonatal rat lung tissues. (a) Tumor necrosis factor-alpha (TNF- $\alpha$ ) immunohistochemistry of lung tissues from each pup group were immunostained with a monoclonal antibody of TNF- $\alpha$ (scale bars $=50 \mu \mathrm{m}$; magnification: $\times 400)$. (b) Quantification of TNF- $\alpha$ immunohistochemistry of lung tissues from each pup group. Values are means \pm SD $(n=8)$. *Significantly different from the time-matched bronchopulmonary dysplasia (BPD) group $(P<0.05)$, ** significantly different from the time-matched BPD group $(P<0.001)$. †Significantly different from the time-matched control group $(P<0.05)$, ${ }^{\text {*}}$ significantly different from the time-matched control group $(P<0.001)$.

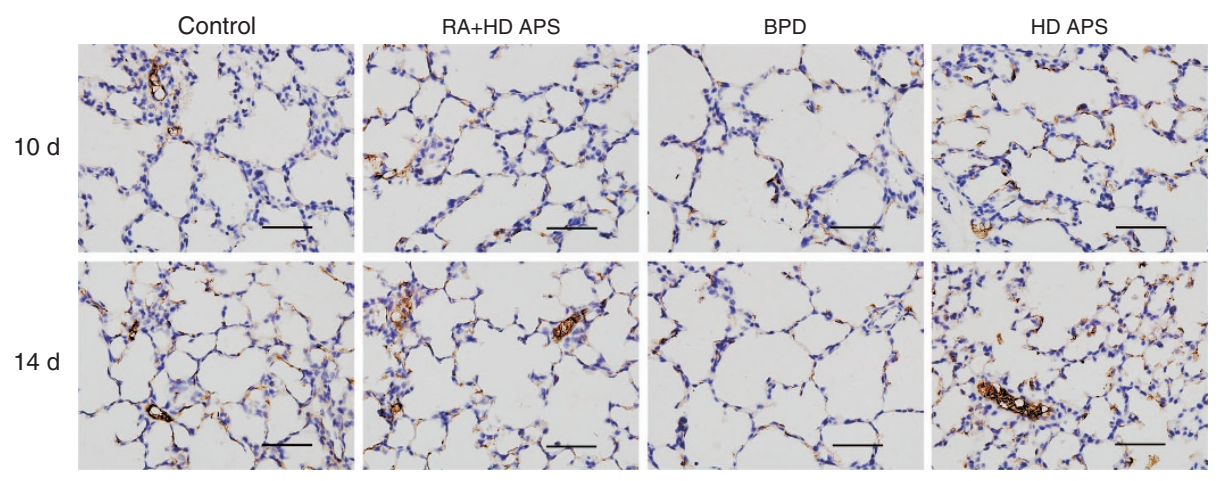

Figure 4. Cell adhesion molecule-1 (CD31) immunohistochemistry. Lung tissues from each pup group were immunostained with a monoclonal antibody of CD31 (scale bars $=50 \mu \mathrm{m}$; magnification: $\times 400$; the low-dose Astragalus polysaccharides group is not shown). 
Table 3. Relative protein levels of CD31 and vascular density of rat lung tissues treated with or without APS

\begin{tabular}{lccccc}
\hline & \multicolumn{2}{c}{ Mean density of CD31 } & & \multicolumn{2}{c}{ Vascular density (\% area) } \\
\cline { 2 - 3 } \cline { 5 - 6 } Group & $10 \mathrm{~d}$ & $14 \mathrm{~d}$ & & $10 \mathrm{~d}$ & $14 \mathrm{~d}$ \\
\hline Control & $0.33 \pm 0.04$ & $0.42 \pm 0.05$ & & $9.58 \pm 1.72$ & $25.39 \pm 3.82$ \\
RA+HD APS & $0.31 \pm 0.05$ & $0.44 \pm 0.14$ & & $9.35 \pm 1.72$ & $25.95 \pm 2.73$ \\
BPD & $0.22 \pm 0.04^{\dagger}$ & $0.22 \pm 0.03^{\ddagger}$ & & $5.06 \pm 0.93^{\ddagger}$ & $14.87 \pm 0.18^{\ddagger}$ \\
HD APS & $0.28 \pm 0.03$ & $0.33 \pm 0.01^{+* *}$ & & $12.22 \pm 0.53^{* *}$ & $28.9 \pm 4.54^{*}$ \\
\hline
\end{tabular}

Values are means \pm SD. Data were analyzed by one-way ANOVA and post hoc NewmanKeuls analysis.

APS, Astragalus polysaccharides; BPD, bronchopulmonary dysplasia; HD APS, highdose Astragalus polysaccharides; RA, room air.

*Significantly different from the time-matched BPD group $(P<0.05)$. * Significantly different from the time-matched BPD group $(P<0.01)$. . Significantly different from the time-matched control group $(P<0.05)$. S Significantly different from the time-matched control group $(P<0.01) ; n=6$ separate samples.

Table 4. Count of neutrophils and macrophages in BALF

\begin{tabular}{lccccc}
\hline & \multicolumn{2}{c}{ Neutrophils $\left(10^{4} / \mathrm{ml}\right)$} & & \multicolumn{2}{c}{ Macrophages $\left(10^{4} / \mathrm{ml}\right)$} \\
\cline { 2 - 3 } \cline { 5 - 6 } Group & $10 \mathrm{~d}$ & $14 \mathrm{~d}$ & & $10 \mathrm{~d}$ & $14 \mathrm{~d}$ \\
\hline Control & $0.86 \pm 0.04$ & $0.80 \pm 0.05$ & & $68.06 \pm 9.06$ & $54.92 \pm 12.85$ \\
RA+HD APS & $0.73 \pm 0.06$ & $0.77 \pm 0.07$ & & $66.29 \pm 18.74$ & $50.41 \pm 3.24$ \\
BPD & $2.34 \pm 0.10^{\ddagger}$ & $2.17 \pm 0.20^{\ddagger}$ & & $117.70 \pm 5.94^{\ddagger}$ & $99.04 \pm 8.28^{\ddagger}$ \\
HD APS & $1.57 \pm 0.05^{* * *}$ & $1.23 \pm 0.29^{* *}$ & $83.61 \pm 7.71^{*}$ & $69.29 \pm 6.27^{* *}$ \\
\hline
\end{tabular}

Values are means \pm SD. Data were analyzed by one-way ANOVA and post hoc NewmanKeuls analysis.

BALF, bronchoalveolar lavage fluid; BPD, bronchopulmonary dysplasia. *Significantly different from the time-matched BPD group $(P<0.05)$. * Significantly different from the time-matched BPD group $(P<0.01)$. 'Significantly different from the time-matched control group $(P<0.05)$. . Significantly different from the time-matched control group $(P<0.01) ; n=8$ separate samples.

d. The western blotting results showed that the protein expressions of NF- $\mathrm{KBp} 65$ and ICAM-1 in the BPD model group were increased compared with the control group at the 4th, 10th, 14 th $\mathrm{d}$, respectively $(P<0.001)$. In the APS groups, the protein expressions of NF- $\mathrm{\kappa Bp} 65$ and ICAM-1 were significantly decreased in a dose-dependent manner compared with the BPD model group (Figure 5). Both protein expressions of NF- $\mathrm{kBp} 65$ and ICAM-1 in RA plus high-dose APS and lowdose APS were not significantly different from the control group at any time point. From the intensities of the histone $\mathrm{H} 3$ and $\beta$-actin bands, we can see that the total protein loading was almost the same in each group.

\section{DISCUSSION}

Previous studies suggest that increasing the SOD activity in the lungs of newborn infants might contribute to preventing inflammatory pulmonary injuries (12). Chen et al. verified that Astragalus could reduce oxidative damage by reduced MDA concentration and increased the activities of SOD and GSHPX, which include scavenging free oxygen radicals (13).

Previous studies have shown that exposure of newborn rats to hyperoxia markedly decreases capillary density when compared with that of air room control (14). In this study, we provided evidence that APS significantly increased the vascular density, reduced lung histological damage, and affected the antioxidant and inflammatory mediators, which result in a remarkable improvement of inflammation injury in BPD rats. Furthermore, SOD increased and MDA decreased in the lung tissues in a dose-time-dependent manner treated with APS. These properties suggest that this Chinese traditional herb Astragalus membranaceus may be useful as both a direct and indirect therapeutic agent for hyperoxia-induced BPD.

Although drugs that target the antioxidant and inflammatory mediators were in wide therapeutic use, they were all identified on the basis of their effect on a specific biological process and were only subsequently shown to target anti-inflammatory cytokines. Recent understanding of the mechanism of antiinflammatory cytokine regulation offers hope for a new generation of drugs on the basis of their ability to modulate the transcription factor nuclear factor $(\mathrm{NF}-\mathrm{\kappa B})$, which might be a potential link between oxidant and inflammatory stress and it regulates the cellular response to inflammatory and oxidant stress (15). It can be activated by hyperoxia, infection, and mechanical ventilation. When NF- $\kappa B$ is activated, then it can subsequently activate transcription of its target genes encoding TNF- $\alpha$ and ICAM-1. Meanwhile, many proinflammatory mediators such as TNF- $\alpha$, ICAM- $1, \mathrm{Cu} / \mathrm{Zn}$ SOD, IL- $1 \beta$, and so on associated with BPD are direct targets of NF- $\mathrm{\kappa B}$ as a shared component among pathways regulating diverse cellular functions, such as differentiation, metabolism, and proliferation (16). Eventually, the inflammatory reaction of the BPD was amplified and perpetuated by the pathogenic cascade (17).

From our immunohistochemistry observation, western blotting analysis, and real-time PCR results, we found that the expressions of NF- $\mathrm{KBp} 65$ and TNF- $\alpha$ were significantly increased in the BPD group and were significantly reduced in the APS group in a dose-dependent manner at both mRNA and protein levels. This suggests that APS may have similar protective effects on BPD with dexamethasone, azithromycin, and so on. In other words, the anti-inflammatory effect of APS may be linked with downregulation in NF- $\mathrm{\kappa Bp} 65$ activity in hyperoxia-induced BPD rats.

TNF- $\alpha$ has been implicated in the pathophysiology of many inflammatory lung diseases, including chronic bronchitis, chronic obstructive pulmonary disease, acute respiratory distress syndrome, and BPD. Elevated levels of TNF- $\alpha$ have been detected in the lungs of various animal and human trials $(5,18,19)$. Besides, elevated TNF- $\alpha$ might be a positive feedback signal that triggered NF- $\kappa \mathrm{B}$ reactivation. It is to be noted that TNF- $\alpha$ stimulates and induces the production of other inflammatory mediators such as ROS, and also activates oxidative stress-responsive genes that amplify and prolong inflammation (20).

In this study, we found that TNF- $\alpha$ markedly increased in the BPD group and significantly reduced in the APS group in a dose-dependent manner in the three given time points which suggested that the APS effect on cytokine levels may be one of its adjunct anti-inflammatory mechanisms. We detected that APS could suppress NF-KBp65 production through inhibited TNF- $\alpha$ in lung tissues (19). It has been reported that APS appears to exert an immune modulating effect by regulating the expression of cytokines such as TNF- $\alpha$ and IL-6 $(21,22)$. 
a

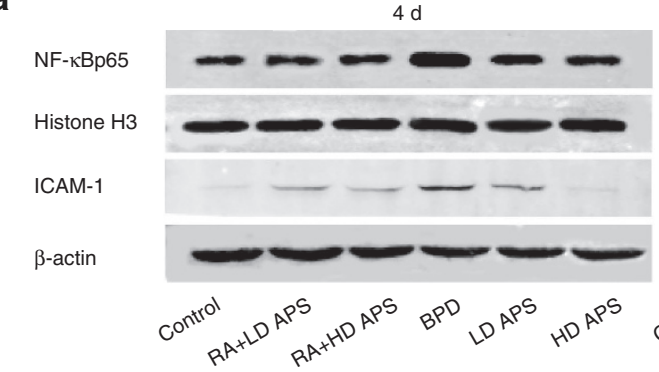

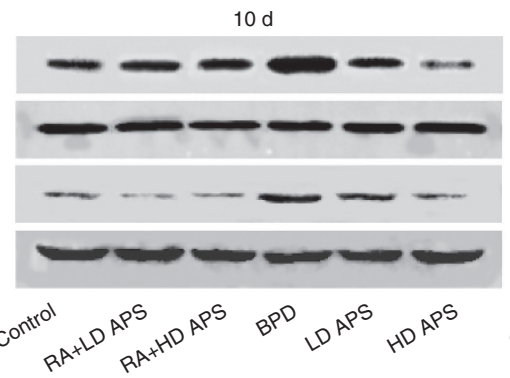

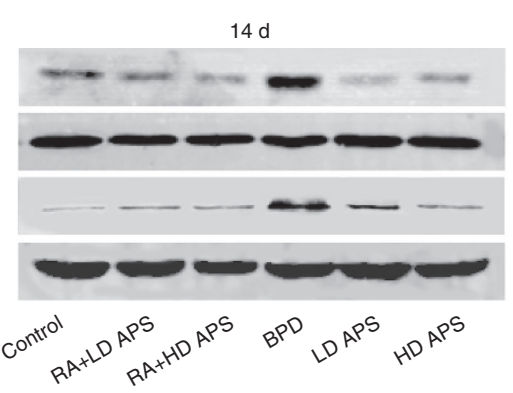

b
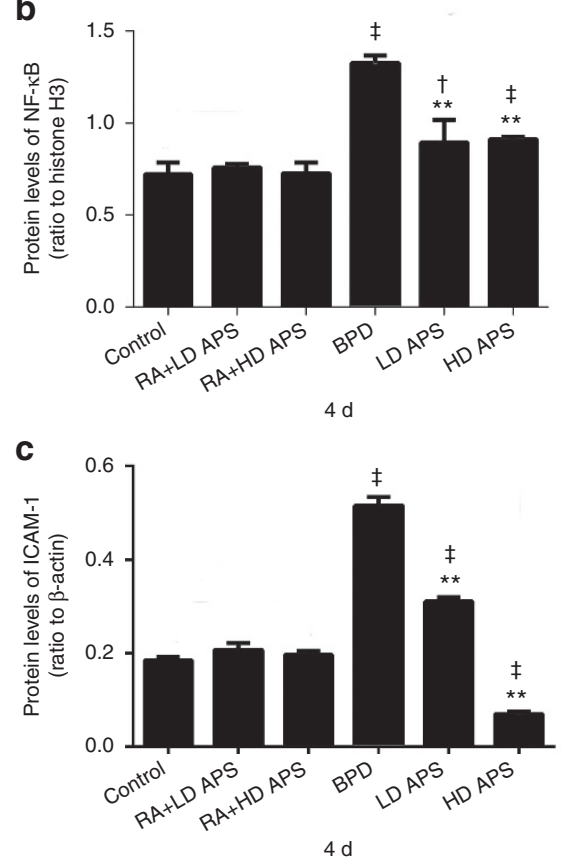
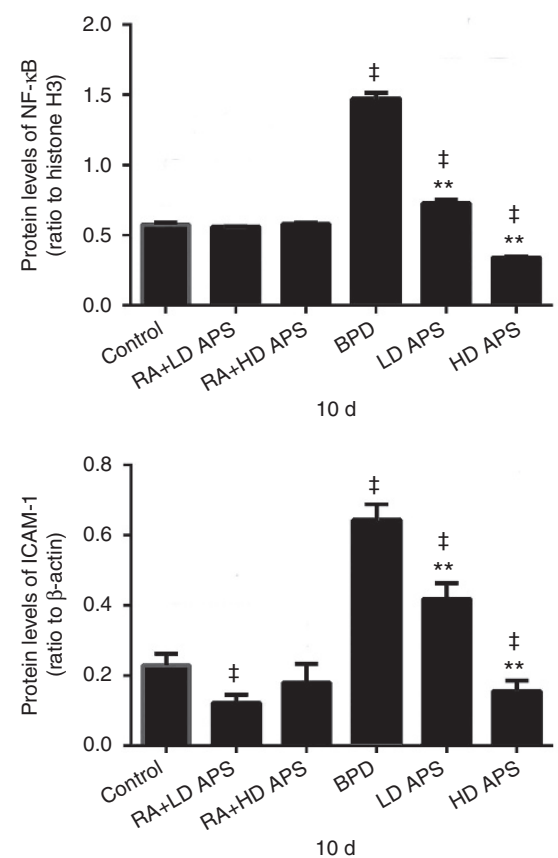
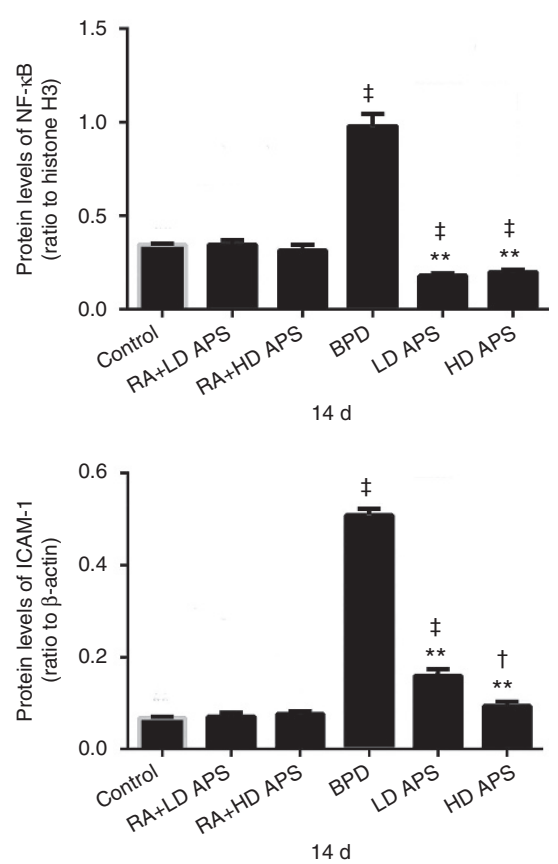

Figure 5. Effects of Astragalus polysaccharides (APS) on ICAM-1 and NF-KBp65 protein expression in neonatal rat lungs. (a) Protein expression of NF-KBp65 and intercellular cell adhesion molecule-1 (ICAM-1) in lung tissues from each pup group identified by western blotting. $\beta$-actin and histone $\mathrm{H} 3$ were used to verify equivalent loading. (b) Quantification of western blotting measured by mean ratios of ICAM-1/ $\beta$-actin and NF- KBp65/histone H3. Protein levels of ICAM-1 of the control group, the room air (RA) plus low-dose APS group, the RA plus high-dose APS group, the bronchopulmonary dysplasia (BPD) model group, the low-dose APS group, and the high-dose APS group. ${ }^{*}$ Significantly different from the time-matched BPD group $(P<0.001)$. (c) Quantification of western blotting measured by mean ratios of NF- $\kappa B p 65 / h i s t o n e ~ H 3$. Protein levels of NF- $\kappa B p 65$ of the control group, the RA plus low-dose APS group, the RA plus high-dose APS group, the BPD model group, the low-dose APS group, and the high-dose APS group. Values are means \pm $\mathrm{SD}(n=8) .{ }^{*}$ Significantly different from the time-matched BPD group $(P<0.001) .{ }^{+}$Significantly different from the time-matched control group $(P<0.05)$, ${ }^{\ddagger}$ significantly different from the time-matched control group $(P<0.001)$.

The ICAM-1, downstream pathway of NF- $\kappa \mathrm{B}$, is a member of the immunoglobulin supergene family which could stimulate neutrophil adhesion and migration and relate to the lung damage in BPD (23). It acted as an early clinical marker for the development of BPD $(24,25)$. Studies indicated that airway concentrations of soluble ICAM- 1 increased at $10 \mathrm{~d}$ of age in infants who develop BPD (26). Our experiment indicated that the levels of ICAM-1 in the BPD group increased more distinctly and declined more obviously in the APS treatment group in response to TNF- $\alpha$, which showed a positive relation with the NF- $\kappa B$ binding activity. This suggests that reduced ICAM-1 by APS might be through modulation of the expression level of NF- $\mathrm{KB}$.

Thus, our results not only contribute to the understanding of the molecular mechanism of APS function in the hyperoxiainduced BPD rats, but also indicate the possible mechanism scavenging oxidative-free radicals, downregulating some proinflammatory mediators and adhesion molecules involved in the immune and inflammatory responses, including TNF- $\alpha$, NF-KBp65, and ICAM-1, which could be a preferred strategy for molecular intervention of pathological processes, such as preventive effects on lung injury.

\section{METHODS}

\section{Animal Experiments}

One hundred and forty-four SD newborn rats $(7.77 \pm 0.56 \mathrm{~g})$ and fungible mother rats were obtained from the Experimental Animal Center of Southern Medical University (Guangzhou, China). This work was approved by the Committee on Animal Ethics and Research at the Southern Medical University. All procedures were conducted according to the Guide for the Care and Use of Laboratory Animals of the US National Institutes of Health (Bethesda, MD). These rats were randomly divided into six groups $(n=24)$ : the control group, the RA plus low-dose APS group, the room air plus high-dose APS group, the BPD 
model group, the low-dose APS group (20mg/kg d), and the high-dose APS group (40 mg/kg d) (SaiNuo Pharmaceutical, Tianjin, China).

The rat model of BPD was induced by hyperoxia. Ten hours after birth, pups in the control group and room air plus APS groups were kept in room air containing $21 \% \mathrm{O}_{2}$, and the latter received daily injections of APS (i.p.) according to the predicted dose throughout the postnatal day, while those in hyperoxia and hyperoxia plus APS groups were placed in an oxygen chamber, into which oxygen was continuously delivered $\left(\mathrm{FiO}_{2}=0.85 \pm 0.03\right)$ and received daily injections of saline (i.p.) and APS (i.p.) according to the predicted dose throughout the postnatal day, respectively. Temperature and humidity were maintained at $22-25^{\circ} \mathrm{C}$ and $60-70 \%$, respectively. The chamber was opened for $1 \mathrm{~h}$ daily to switch dams between air and $\mathrm{O}_{2}$ environment to protect the dams from oxygen toxicity. These pups were then killed at the 4 th, 10th, and 14th d after experiments and the lung tissues were collected.

\section{Assessment of Lung Histological Damage}

Following anesthesia with pentobarbital $(60 \mathrm{mg} / \mathrm{kg}$, i.p.), the left lungs of pups were fixed with an intratracheal injection of $4 \%$ paraformaldehyde at a constant pressure of $20 \mathrm{~cm} \mathrm{H}_{2} \mathrm{O}$ and postfixed in $4 \%$ paraformaldehyde overnight at room temperature. The left lung volume $\left(V_{\mathrm{L}}\right)$ was measured by fluid displacement (27). Tissues were paraffin embedded, sectioned to $5-\mu \mathrm{m}$ thickness, and stained with hematoxylin and eosin. Quantitative analysis of pulmonary MLI and MAN was measured according to previously described methods (28).

\section{Lung SOD Activity and MDA Level Assays}

The right lung tissues were rinsed and weighed, then put into tubes with the corresponding volumes of $0.86 \mathrm{~g} / \mathrm{l}$ normal saline, according to the weight $(\mathrm{g})$ :volume $(\mathrm{ml})=1: 9$ proportion. Then the tissue samples were homogenized for $10 \mathrm{~min}$. After centrifugation at 2,500 rpm for $15 \mathrm{~min}$ at $4{ }^{\circ} \mathrm{C}$, the MDA contents and SOD activities in the supernatant were measured by the assay kit (Nanjing Jiancheng, Nanjing, China) according to its provider's instructions.

\section{RNA Extraction Real-Time Fluorescent Quantitative PCR}

Total RNA from rat lungs was isolated using the Trizol kit (TaKaRa Bio, Dalian, Liaoning, China) following the manufacturer's protocol. Singlestrand cDNA synthesis was carried out using $2 \mathrm{mg}$ of total RNA by the reverse transcription (RT) reaction using the PrimeScript RT reagents Kit (TaKaRa Bio). Real-time PCR was conducted using the Applied Biosystems 7500 Real Time PCR System (Life Technologies, Carlsbad, $\mathrm{CA}$ ) in the reactions containing SYBR Premix Ex Taq (Tli RNaseH Plus) Kit (TaKaRa Bio) mix reagent and oligonucleotide pairs to the endogenous control $\beta$-actin gene, NF- $\kappa B p 65$, TNF- $\alpha$, ICAM1-1, and cDNA. After being matched with oligo dT and reverse transcribed to cDNA, it was then primed with oligo $\mathrm{d} T$ and subsequently amplified using specific oligonucleotide primers. The primers were synthesized by Invitrogen Biotech (Shanghai, China). Two microliters of cDNA was amplified in $20 \mu \mathrm{l}$ of PCR solution. Thermal cycling parameters consisted of $95^{\circ} \mathrm{C}$ for $30 \mathrm{~s}$, followed by 40 cycles of $95^{\circ} \mathrm{C}$ for $5 \mathrm{~s}$ and $60^{\circ} \mathrm{C}$ for $34 \mathrm{~s}$. The primer sequences $\left(5^{\prime}-3^{\prime}\right)$ were as follows:

\section{NF- $\kappa$ Bp65 forward: 5'CCGGGATGGCTTCTATGAG3';

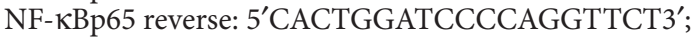 \\ TNF- $\alpha$ forward: 5'TGAAGTAGTGGCCTGGATTGC3'; \\ TNF- $\alpha$ reverse: $5^{\prime}$ GACATTCCGGGATCCAGTGA3'; \\ ICAM1-1 forward: 5'CTTTAGCAGCTCAACAATGG3'; \\ ICAM1-1 reverse: 5'CATTTTCTCCCAGGCATTC3'; \\ $\beta$-actin forward: 5'AGGGAAATCGTGCGTGACAT3'; \\ $\beta$-actin reverse: 5'GAACCGCTCATTGCCGATAG3'.}

Average CT values for NF- $\kappa B p 65$, TNF- $\alpha$, and ICAM1-1 were calculated and normalized to CT values for $\beta$-actin, and the normalized values were subjected to a $2^{-\Delta \Delta C T}$ formula to calculate the fold change between the control and experiment groups. The formula and its derivations were obtained from the Applied Biosystems 7500 Real Time PCR System user guide. All reactions were performed in triplicate.

\section{Immunohistochemistry}

Left lung tissues obtained at each time point were fixed with an intratracheal injection of $4 \%$ paraformaldehyde and postfixed overnight at room temperature. Tissues were then paraffin embedded, sectioned to $5-\mu \mathrm{m}$ thickness, and deparaffinized in xylene and hydrated in a series of graded alcohol. After dewaxing and rehydration, sections were immersed in 3\% hydrogen peroxide in methanol for $20 \mathrm{~min}$ at room temperature to abolish endogenous peroxidase activities and then the antigen retrieval was done by microwave for $15 \mathrm{~min}$ before they were blocked with $5 \%$ bovine serum albumin (Life Technologies, Carlsbad, California) at $37^{\circ} \mathrm{C}$ for $20 \mathrm{~min}$. Slides were incubated with mouse polyclonal antibody of TNF- $\alpha$ (1:50, Abcam Biotechnology, Cambridge, $\mathrm{UK}$ ) and mouse monoclonal CD31 antibody (1:200, Novus Biologicals, Littleton, CO) at $37^{\circ} \mathrm{C}$ for $2 \mathrm{~h}$. After PBS washing, the slides were incubated with a biotinylated peroxidase-conjugated secondary antibody and $0.1 \% \mathrm{DAB}$ substrate, using the standard streptavidin-biotin-based method. Immunoadsorption was employed as the negative control. Specific primary antibodies were first incubated with a corresponding peptide immunogen (TNF- $\alpha$ and CD31 (Bioworld Technology, St Louis Park, MN), respectively) to block antibody binding sites, and then this mixture was subsequently applied to tissue sections. A cytoplasmic brown granule was marked as a positive expression of TNF- $\alpha$ and $\mathrm{CD} 31$. We quantified vascular density by measuring the area of CD31 immunostaining relative to the total area of parenchymal cells using Image-Pro Plus 6.0 (Media Cybernetics, MD) of differential interference contrast images. Six random fields were chosen from each slide for five animals at each time point. Images from each slide were captured with a $400 \times$ objective. The tissue sections analyzed contained mainly the alveolar parenchyma and excluded any large airways or blood vessels. The intensities of the TNF- $\alpha$ expression were analyzed by ImagePro Plus 6.0 too, and mean density was measured.

\section{Preparation of Bronchoalveolar Lavage Fluid}

Bronchoalveolar lavage fluid was collected on the 4 th, 10th, and 14th $\mathrm{d}$ by using three consecutive instillations of PBS ( $0.5 \mathrm{ml}$ each) at room temperature. Approximately $1.2 \mathrm{ml}$ of the PBS was consistently recovered with gentle handling and centrifuged at $500 \mathrm{~g}$ at $4{ }^{\circ} \mathrm{C}$ for $5 \mathrm{~min}$. Cell pellets were resuspended in $0.5 \mathrm{ml} \mathrm{PBS}$ and smeared for analysis of cellular infiltration. Cellular infiltration in bronchoalveolar lavage fluid was assessed by Wright's stain.

\section{Western Blotting}

Protein was extracted from SD rat lung tissues of different groups with lysis buffer using the NE-PER Nuclear and Cytoplasmic Extraction Reagent Kit (Thermo Fisher Scientific, Rockford, IL). The protein concentration of each sample was measured by the Keygen BCA Protein Assay (Keygen, Nanjing, China). $40 \mu \mathrm{g} / \mathrm{lane}$ of each cytosolic and nuclear extract sample was analyzed by $10 \%$ SDS-PAGE and transferred to PVDF membranes. Membranes were blocked in 5\% nonfat milk in TBS $+0.1 \%$ Tween 20, and incubated with monoclonal antiNF-אBp65, anti-ICAM-1 (Signalway Antibody, College Park, MD) diluted at 1:500 and anti-histone H3 (Bytotime Biotechnology, Jiangsu, China), anti- $\beta$-actin (ZSGB-Bio, Beijing, China) used as the nuclear and cytoplasmic endogenous control, respectively, both diluted at 1:2,000. Anti-rabbit $(\mathrm{H}+\mathrm{L}) \mathrm{HRP}$ and anti-mouse $(\mathrm{H}+\mathrm{L})$ HRP diluted at 1:2,000 was used as the secondary antibody with SuperSignal West Pico Chemiluminescent Substrate (Thermo Fisher Scientific, Rockford, IL) for detection. The membrane was further incubated for $90 \mathrm{~min}$ at 37 ${ }^{\circ} \mathrm{C}$. The intensities of the protein bands were analyzed by Quantity One (Bio-Rad Laboratories, Hercules, CA).

\section{Statistical Analysis}

The results were expressed as mean \pm SD. Statistical analysis was subjected to one-way ANOVA and the Student-Newman-Keuls test with factors of treatment and disease or normal type by SPSS 13.0 statistical software (SPSS, Chicago). A value of $P<0.05$ was considered significantly different.

\section{STATEMENT OF FINANCIAL SUPPORT}

This work was supported by the Guangdong Province Science and Technology Plan Project (2012B031800303 to W.M.H.), the National Clinical Key Specialty Project Foundation (L.D.), and the Guangdong Natural Science Foundation ( 9151008901000033 to L.D.). 


\section{Articles | Wang et al.}

\section{REFERENCES}

1. Fernandez-Gonzalez A, Alex Mitsialis S, Liu X, Kourembanas S. Vasculoprotective effects of heme oxygenase-1 in a murine model of hyperoxiainduced bronchopulmonary dysplasia. Am J Physiol Lung Cell Mol Physiol 2012;302:L775-84.

2. Allen Merritt T, Deming DD, Boynton BR. The 'new' brochopulmonary dysplasia: challenges and commentary. Semm Fetal Neonatal Med 2009;14:345-57.

3. Köksal N, Kayik B, Çetinkaya M, et al. Value of serum and bronchoalveolar fluid lavage pro- and anti-inflammatory cytokine levels for predicting bronchopulmonary dysplasia in premature infants. Eur Cytokine Netw 2012;23:29-35.

4. Tieri P, Termanini A, Bellavista E, Salvioli S, Capri M, Franceschi C. Charting the NF-KB pathway interactome map. PLoS One 2012;7:e32678.

5. Speer CP. Chorioamnionitis, postnatal factors and proinflammatory response in the pathogenetic sequence of bronchopulmonary dysplasia. Neonatology 2009;95:353-61.

6. Soll RF. Corticosteroids for the treatment and prevention of bronchopulmonary dysplasia. Neonatology 2010;98:109-10.

7. Chu C, Qi LW, Liu EH, Li B. Radix Astragali (Astragalus): latest advancements and trends in chemistry, analysis, pharmacology and pharmacokinetics. Curr Org Chem 2010;14:1792-807

8. Gülbayzar S, Arica V, Hatipoğlu S, Kaya A, Arica S, Karatekin G. Malondialdehyde level in the cord blood of newborn infants. Iran J Pediatr 2011;21:313-9.

9. Forman HJ, Fridovich I. Superoxide dismutase: a comparison of rate constants. Arch Biochem Biophys 1973;158:396-400.

10. Tin W, Wiswell TE. Drug therapies in bronchopulmonary dysplasia: debunking the myths. Semin Fetal Neonatal Med 2009;14:383-90.

11. Davis JM, Rosenfeld WN, Sanders RJ, Gonenne A. Prophylactic effects of recombinant human superoxide dismutase in neonatal lung injury. J Appl Physiol (1985) 1993;74:2234-41.

12. Davis JM. Role of oxidant injury in the pathogenesis of neonatal lung disease. Acta Paediatr Suppl 2002;91:23-5.

13. Chen R, Shao H, Lin S, Zhang JJ, Xu KQ. Treatment with Astragalus membranaceus produces antioxidative effects and attenuates intestinal mucosa injury induced by intestinal ischemia-reperfusion in rats. Am J Chin Med 2011;39:879-87.

14. Shaffer SG, O’Neill D, Bradt SK, Thibeault DW. Chronic vascular pulmonary dysplasia associated with neonatal hyperoxia exposure in the rat. Pediatr Res 1987;21:14-20.
15. Wright CJ, Kirpalani H. Targeting inflammation to prevent bronchopulmonary dysplasia: can new insights be translated into therapies? Pediatrics 2011;128:111-26.

16. Perkins ND. Integrating cell-signalling pathways with NF-kappaB and IKK function. Nat Rev Mol Cell Biol 2007;8:49-62.

17. Gien J, Kinsella JP. Pathogenesis and treatment of bronchopulmonary dysplasia. Curr Opin Pediatr 2011;23:305-13.

18. Bose CL, Dammann CE, Laughon MM. Bronchopulmonary dysplasia and inflammatory biomarkers in the premature neonate. Arch Dis Child Fetal Neonatal Ed 2008;93:F455-61.

19. Wright CJ, Agboke F, Chen F, LA P, Yang G, Dennery PA. NO inhibits hyperoxia-induced NF- $\mathrm{KB}$ activation in neonatal pulmonary microvascular endothelial cells. Pediatr Res 2010;68:484-9.

20. Larrick JW, Wright SC. Cytotoxic mechanism of tumor necrosis factoralpha. FASEB J 1990;4:3215-23.

21. He X, Shu J, Xu L, Lu C, Lu A. Inhibitory effect of Astragalus polysaccharides on lipopolysaccharide-induced TNF- $\alpha$ and IL-1 $\beta$ production in THP-1 cells. Molecules 2012;17:3155-64.

22. Yuan Y, Sun M, Li KS. Astragalus mongholicus polysaccharide inhibits lipopolysaccharide-induced production of TNF-alpha and interleukin-8. World J Gastroenterol 2009;15:3676-80.

23. Hang CH, Shi JX, Li JS, Wu W, Yin HX. Concomitant upregulation of nuclear factor- $\mathrm{KB}$ activity, proinflammatory cytokines and ICAM-1 in the injured brain after cortical contusion trauma in a rat model. Neurol India 2005;53:312-7.

24. Kojima T, Sasai M, Kobayashi Y. Increased soluble ICAM-1 in tracheal aspirates of infants with bronchopulmonary dysplasia. Lancet 1993;342:10234 .

25. Ramsay PL, O’Brian Smith E, Hegemier S, Welty SE. Early clinical markers for the development of bronchopulmonary dysplasia: soluble E-Selectin and ICAM-1. Pediatrics 1998;102(4 Pt 1):927-32.

26. Kotecha S, Chan B, Azam N, Silverman M, Shaw RJ. Increase in interleukin-8 and soluble intercellular adhesion molecule-1 in bronchoalveolar lavage fluid from premature infants who develop chronic lung disease. Arch Dis Child Fetal Neonatal Ed 1995;72:F90-6.

27. Scherle W: A simple method for volumetry of organs in quantitative stereology. Mikroskopie 1970;26:57-60.

28. Robbesom AA, Versteeg EM, Veerkamp JH, et al. Morphological quantification of emphysema in small human lung specimens: comparison of methods and relation with clinical data. Mod Pathol 2003;16: $1-7$. 